\title{
Testing the actual equivalence of automatically generated items
}

\author{
Debora de Chiusole $^{1} \cdot$ Luca Stefanutti $^{1} \cdot$ Pasquale Anselmi $^{1} \cdot$ Egidio Robusto $^{1}$ \\ Published online: 16 January 2018 \\ (C) Psychonomic Society, Inc. 2018
}

\begin{abstract}
If the automatic item generation is used for generating test items, the question of how the equivalence among different instances may be tested is fundamental to assure an accurate assessment. In the present research, the question was dealt by using the knowledge space theory framework. Two different ways of considering the equivalence among instances are proposed: The former is at a deterministic level and it requires that all the instances of an item template must belong to exactly the same knowledge states; the latter adds a probabilistic level to the deterministic one. The former type of equivalence can be modeled by using the BLIM with a knowledge structure assuming equally informative instances; the latter can be modeled by a constrained BLIM. This model assumes equality constraints among the error parameters of the equivalent instances. An approach is proposed for testing the equivalence among instances, which is based on a series of model comparisons. A simulation study and an empirical application show the viability of the approach.
\end{abstract}

Keywords Knowledge space theory $\cdot$ Basic local independence model $\cdot$ Knowledge assessment $\cdot$ Automatic item generation · Item template

\section{Introduction}

Web-based computerized tests for individual knowledge assessment offer many advantages as compared to more traditional paper-and-pencil tests. For example, they provide examinees with instant feedback and they allow recurring and adaptive assessments. Nevertheless, they also raise new challenges, particularly in the items development: Large item banks are needed because items are continuously administered and, therefore, exposed (Gierl \& Haladyna, 2013).

Traditional item development requires experts to write items one by one, leading to a very expensive and time-consuming task (Rudner, 2010). Furthermore, each single new item must be empirically tested. Automatic item generation

Debora de Chiusole

debora.dechiusole@unipd.it

Luca Stefanutti

luca.stefanutti@unipd.it

Pasquale Anselmi

pasquale.anselmi@unipd.it

Egidio Robusto

egidio.robusto@unipd.it

1 University of Padua (Italy), FISPPA Department Via Venezia, 14, 35131 Padua, Italy
(AIG; Arendasy \& Sommer 2012; Embretson \& Yang 2006; Gierl \& Haladyna 2013; Irvine \& Kyllonen 2013; Bormuth 1969) is an alternative method for producing test items, consisting in outlining an "item template", from which a very huge, theoretically infinite, number of instances is generated.

Assuming that a particular method for developing a certain number of instances from the same item template exists, the question remains open of how the equivalence among different instances may be tested. For answering this question, the very first step is to provide a definition of instance equivalence. From a behavioral perspective, two or more instances are "equivalent" in a population of subjects if the answers, either correct or incorrect, of an individual to the instances are the same, excluding measurement errors. If so, the instances are interchangeable and they can be used in the assessment, indifferently. The aim of the present research is to provide a procedure for testing the equivalence of two or more instances, with this meaning.

The idea of developing parallel tests for the assessment of the same psychological variable was born in classical test theory (CTT) for the assessment of cognitive skills in the so-called "mental tests". In this approach, in order to test the equivalence among two or more parallel forms of the same test, some statistical methods were developed. These methods are based on verifying if the means, variances and covariances of test scores are equal for different parallel forms. 
For instance, the reader can refer to Gulliksen (2013) for a review of these methods.

A different approach for building psychological assessment tests is the well-known item response theory (IRT). This theory is successfully applied to knowledge assessment, in order to estimate both the person ability and the item difficulty. In IRT, some statistical and psychometric models have been suggested for testing the equivalence of the so-called item clones (Glas and van der Linden, 2003), structurally equivalent items or also schemata (Singley \& Bennett, 2002). To give an example, one of these models is the 2PL-constrained model (C2PL; Embretson 1999, 2005). One of its peculiarities is that it predicts the psychometric properties of the instances from the so-called "design system" properties used for generating them, so that it allows the calibration of the design principle rather than the calibration of specific instances. For an overview of this and other statistical models for testing automatically generated items, see Sinharay and Johnson (2013).

Both CTT and IRT approaches assume unidimensionality. Furthermore, they both consider the psychological variables as continuous. In the knowledge assessment area, which is the framework of the present research, neither unidimensionality nor continuity are assumed.

In the last four decades, two knowledge-assessment approaches have been developed and successfully applied: The knowledge space theory (KST; Albert \& Lukas 1999; Doignon \& Falmagne 1999; Falmagne \& Doignon 2011) and the cognitive diagnostic models (CDMs; Bolt 2007; DiBello \& Stout 2007; Junker \& Sijtsma 2001; Tatsuoka 1990). The reader is referred to Heller et al. (2015) and Heller et al. (2016) for the connection between these two theories. CDMs can be used when the interest is to estimate a student's skill profile.

Concerning KST, it is a mathematical theory for the efficient assessment of knowledge, started in the 1980s by Doignon and Falmagne (1985); See also Doignon and Falmagne (1999) and Falmagne and Doignon (2011). In this theory, the main goal of the assessment is to identify the knowledge state of a student, which is the set of problems that he is able of solving in a certain knowledge domain.

Both CDMs and KST use software implementing online automatic item generation for producing equivalent instances of a test item. So that, during a computerized adaptive assessment, different instances generated from the same item template are used indifferently. In CDMs area, several methods for testing the homogeneity of the instances and of the tests exist, like the classification consistency and the classification accuracy (Cui et al., 2012). Some statistical methods were developed and applied to real data also in KST. These methods were developed with the aim of evaluating the reliability/validity of an adaptive assessment.
They are based on the administration of an "extra problem" (item). The extra problem is randomly selected from the set of all the problems that were not administered to the student, and it is presented at the end of the assessment, once the student's knowledge state has already been estimated. The response (correct or incorrect) of the student to this problem is then compared to the one predicted by the estimated knowledge state. The validity of the prediction is evaluated by standard measures of correlation, as tetrachoric, phicorrelation, and point biserial coefficients. Nevertheless, as pointed out by the authors themselves, these statistical indexes have serious drawbacks (Cosyn et al., 2013).

All the methods described above addressed the problem of testing the equivalence among instances by using statistical indexes of correlation. Although using these indexes seems the most natural way for testing instance equivalence, in the theoretical study presented in "A naïve method for testing instance equivalence", it is shown that they are strongly affected by the probabilities of the instances. This was one of the reasons that led us to tackle the issue by using a modeling approach.

In this article, we propose two different ways of considering the equivalence among instances: The former is at a deterministic level and it requires that all the instances of an item template must belong to exactly the same knowledge states; The latter adds a probabilistic level to the deterministic one, by introducing equality constraint assumptions regarding the error parameters of the instances. This distinction gives rise to two different ways of modeling the instance equivalence. The former type of equivalence can be modeled, for example, by using the basic local independence model (BLIM; Falmagne \& Doignon 1988a, b) with a knowledge structure in which the instances belong to exactly the same states of the structure. The latter can be modeled by a special case of the BLIM, in which parameter equality constraints are introduced.

Thus, in "Background" of the paper, some backgrounds needed in the subsequent sections are given. In "A naïve method for testing instance equivalence", a naïve method for testing instance equivalence is presented. In "Modeling instance equivalence in KST", after introducing the two definitions of instance equivalence, the two ways of modeling it are presented and the constrained BLIM model is derived. Then, the two proposed methods are compared in both a simulation study ("Simulation study") and an empirical application ("Empirical application"). The aim was to study their effect on the equivalence of the instances generated from item templates assessing knowledge of the "descriptive statistics" and "probability theory" topics.

A general discussion of the results ("Conclusions") concludes the argumentation and some hints for the item templates construction are given. 


\section{Background}

KST is a mathematical theory for the efficient assessment of knowledge (Doignon \& Falmagne 1985, 1999; Falmagne \& Doignon 2011). In this theory, the main goal of the assessment is to identify the knowledge state of a student, which is formally defined as the subset $K$ of problems $q$ in a given field $Q$ of knowledge, that the student is capable of solving. The collection $\mathcal{K}$ of all the knowledge states existing in a population of students is called knowledge structure.

A key concept used in this article is that of equally informative items (Doignon \& Falmagne, 1999), namely items that belong to exactly the same knowledge states. The notion of equally informative items induces an equivalence relation $\sim$ on the set $Q$. Indicating with $\mathcal{K}_{q}=\{K \in \mathcal{K}$ : $q \in K\}$ the collection of all the states containing a certain item $q \in Q$, the equivalence relation is defined by:

$q \sim r \Longleftrightarrow \mathcal{K}_{q}=\mathcal{K}_{r}$,

for all $q, r \in Q$. Thus, the equivalence class of an item $q$

$q^{*}=\{r \in Q: r \sim q\}$,

is called notion. It follows that every item template is a notion $q^{*} \in Q^{*}$. Moreover, let $Q^{*}=\left\{q^{*}: q \in\right.$ $Q\}$ denote the collection of all the equivalence classes, that is the quotient set on $Q$. A knowledge structure in which each notion is a singleton is called discriminative. In general, every knowledge structure can be converted into a discriminative one. Given a knowledge structure $\mathcal{K}$ on the set $Q$, its discriminative reduction is the pair $\left(Q^{*}, \mathcal{K}^{*}\right)$ with

$\mathcal{K}^{*}=\left\{K^{*}: K \in \mathcal{K}\right\}$,

where for any $K \in \mathcal{K}$, we have $K^{*}=\left\{q^{*}: q \in K\right\}$. Therefore, $\mathcal{K}$ is a knowledge structure on the set $Q$ of item instances, whereas $\mathcal{K}^{*}$ is a knowledge structure on the set $Q^{*}$ of item templates.

A knowledge structure is a deterministic model of the organization of knowledge that requires an appropriate probabilistic treatment to be empirically validated. The BLIM is the most used probabilistic model for knowledge structure. It is characterized by the following main aspects:

1. Given a population of students, some probability distribution on the collection of knowledge states exists. Formally a probabilistic knowledge structure is introduced, which is a triple $(Q, \mathcal{K}, \pi)$, where $(Q, \mathcal{K})$ is a knowledge structure and $\pi$ is a probability distribution on $\mathcal{K}$ (i.e., $\pi_{K} \geq 0$ for all $K \in \mathcal{K}$ and $\sum_{K \in \mathcal{K}} \pi_{K}=1$ );

2. A distinction is made between the (unobservable) knowledge state $K \in \mathcal{K}$ of a student and his observable response pattern $R \subseteq Q$, that is the collection of all the problems that received a correct answer by the student. The relationship between $K$ and $R$ is given by the unrestricted latent class model

$P(R)=\sum_{K \in \mathcal{K}} P(R \mid K) \pi_{K}$,

where $P(R)$ is the probability of sampling a student whose response pattern is $R$, and $P(R \mid K)$ is the conditional probability of observing response pattern $R$ given that the knowledge state is $K$;

3. An assumption is introduced concerning the conditional probabilities $P(R \mid K)$ in Eq. 1. This assumption is called response rule and states that the conditional probability of obtaining a correct response for an item $q$, given a certain knowledge state $K \in \mathcal{K}$, is given by two parameters: $\beta_{q} \in[0,1)$, that is the conditional probability of observing an incorrect answer to item $q$ given that $q \in K$, and $\eta_{q} \in[0,1)$, that is the conditional probability of observing a correct answer to item $q$ given that $q \notin K$. The $\beta_{q}$ and $\eta_{q}$ parameters are called careless error and lucky guess, respectively;

4. Under the response rule assumption and the assumption of local independence of the item responses, given the knowledge states, the conditional probability $P(R \mid K)$ of a response pattern $R \subseteq Q$, given a knowledge state $K \in \mathcal{K}$, takes on the form

$P(R \mid K)=\left[\prod_{q \in K \backslash R} \beta_{q}\right]\left[\prod_{q \in K \cap R}\left(1-\beta_{q}\right)\right]\left[\prod_{q \in R \backslash K} \eta_{q}\right]\left[\prod_{q \in Q \backslash(R \cup K)}\left(1-\eta_{q}\right)\right]$.

Concerning the BLIM, methods for estimating its parameters (Heller \& Wickelmaier, 2013; Schrepp, 2005; Stefanutti $\&$ Robusto, 2009) and for testing its identifiability (Spoto et al., 2012; Stefanutti et al., 2012) are available, together with methods for testing its assumption about the parameter invariance (de Chiusole et al. 2013, 2015b). Furthermore, several extensions of the BLIM have been proposed, which allow for modeling skill dependencies (de Chiusole \& Stefanutti, 2013), as well as for treating missing data (de Chiusole et al., 2015a; Anselmi et al., 2016). There is also a model for assessing learning processes (Robusto et al., 2010; de Chiusole et al., 2013; Anselmi et al., 2017). 


\section{A naïve method for testing instance equivalence}

In order to test the equivalence of two instances $q, r \in$ $Q$, the most natural way might be of correlating the responses observed for $q$ with those observed for $r$. If the two instances are in effect equivalent, then one would expect a high value of correlation. Given the dichotomous nature of the responses (correct or wrong) to the test items in the knowledge assessment area, standard measures of correlation between two dichotomous variables can be used. One of these types of measure is the $\phi$-coefficient.

At this point, the question raises if the $\phi$-coefficient is adequate to correctly discover the equivalence of the instances, if it exists. Thus, a theoretical study was carried out for examining, in particular, the effect on the $\phi$ coefficient of (a) the instance probabilities $P(q, r)=$ $\sum_{K \in \mathcal{K}_{q} \cap \mathcal{K}_{r}} \pi_{K}$, where $\mathcal{K}_{q}$ and $\mathcal{K}_{r}$ are the collections of states $K \in \mathcal{K}$ containing items $q$ and $r$, respectively; and of (b) the error probability of the instances (e.g., the parameters $\beta_{q}, \eta_{q}, \beta_{r}$ and $\eta_{r}$ estimated by the BLIM). The study is theoretical because the $\phi$ coefficient was computed by using the expected frequencies of the response patterns predicted by the BLIM, thus obtaining an expected value of the $\phi$ coefficient.

\section{Methods}

The $\phi$ coefficient was computed by an application of the following formula:

$\phi=\frac{p(q, r)-p(q) p(r)}{\sqrt{p(q) p(r)(1-p(q))(1-p(r))}}$,

where $p(q, r)$ is the probability of observing a response pattern containing both items $q$ and $r, p(q)$ and $p(r)$ are the marginal probabilities of items $q$ and $r$, respectively. These three probabilities are predicted by the BLIM. It should be noted that $p(q, r)$ is the probability that $q$ and $r$ belong to the same response pattern, whereas $P(q, r)$ is the probability of a knowledge state containing both $q$ and $r$.

Ten different values of careless error and luckyguess probabilities and twenty different values of instance probabilities were used for computing the expected $\phi$ coefficient. In order to facilitate comparisons, the equality constraint $\beta_{q}=\beta_{r}=\eta_{q}=\eta_{r}$ was used. The values of these parameters were equally spaced points in the closed interval $[.01, .45]$, whereas the values of the instance probabilities $P(q, r)$ were equally spaced points in the closed interval $[0,1]$.

\section{Results and discussion}

Figure 1 displays the expected $\phi$ coefficient as a function of the instance probabilities $P(q, r)$. Each curve in the diagram corresponds to a different value of the probabilities $\beta_{q}=\beta_{r}=\eta_{q}=\eta_{r}$ used for computing $\phi$.

It is clear that the $\phi$ coefficient is strongly affected by both the instance probabilities $P(q, r)$ and the item error parameters. Concerning the error parameters, the higher their values the lower the $\phi$ coefficient. Concerning the instance probabilities, $\phi$ approaches zero as $P(q, r)$ moves away from .50 .

These are clear drawbacks of the $\phi$ coefficient. On the one side, a low value of this coefficient could be a mere result of high careless errors and lucky-guess probabilities, even when the items are indeed equally informative. On the other side, a low value of this coefficient could be an effect of low or high values of the instance probabilities. With the error set to .01 , as the instance probability varies from .10 to 0 , the $\phi$ coefficient rapidly decreases from .90 to 0 .

Thus, we conclude that the interpretation of the $\phi$ coefficient is ambiguous because, even when the instances are equally informative, this index could be very low.

\section{Modeling instance equivalence in KST}

In KST, at least two different definitions of instance equivalence can be given. The former is at a deterministic level, whereas the latter is at both a deterministic level and a probabilistic level.

According to the first definition, given a knowledge structure $(Q, \mathcal{K})$, two instances $q, r \in Q$ of an item template are equivalent if and only if they belong to exactly the same states in $\mathcal{K}$.

Besides equal informativeness, the second definition of instance equivalence requires that instances have the same careless error and lucky guess parameters. Formally:

$q \sim r \Longrightarrow \beta_{q}=\beta_{r}$ and $\eta_{q}=\eta_{r}$

for all $q, r \in Q$.

The distinction between the two definitions gives rise to two different ways of modeling instance equivalence. The former type of equivalence can be easily modeled by a direct application of the BLIM to the knowledge structure $\mathcal{K}$ on the set $Q$ of all item the instances. It should be noted that, in this model, equally informative items may have different careless error and lucky guess probabilities. Therefore, the BLIM is not suitable for testing the second type of equivalence. However, the BLIM can be readily 


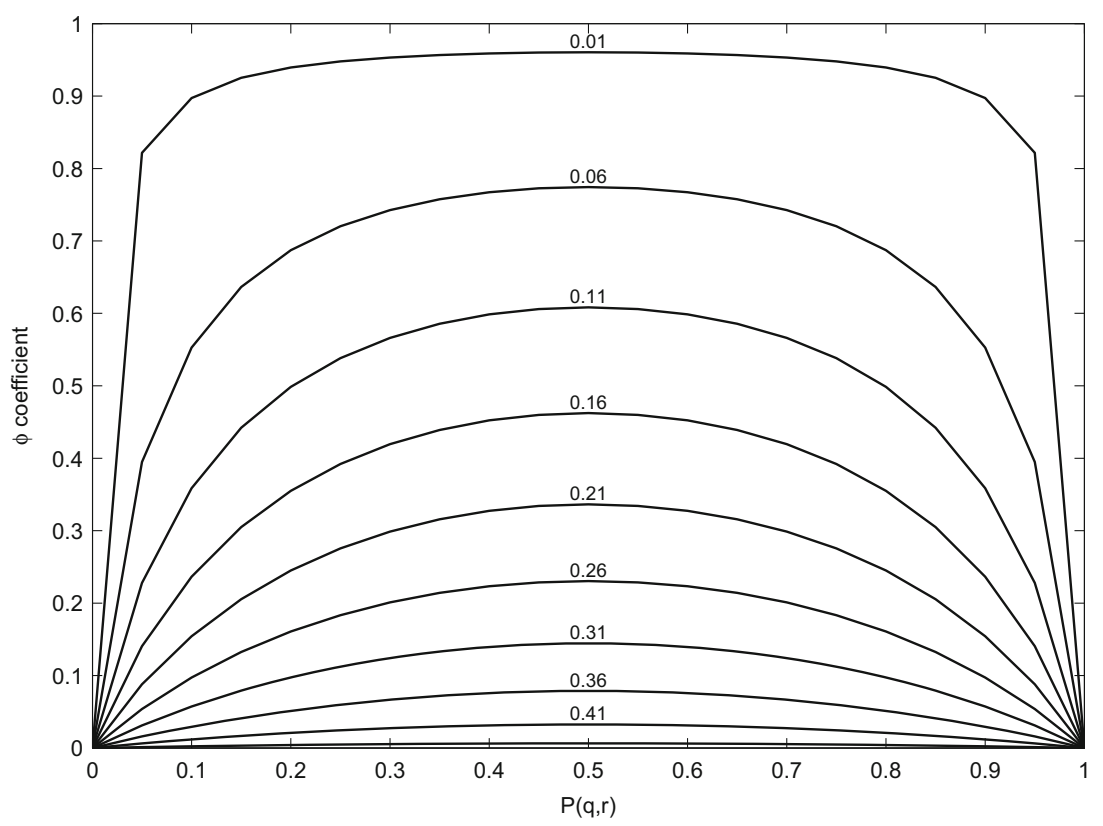

Fig. 1 Expected $\phi$ coefficient as a function of the instance probabilities $P(q, r)$. Each curve in the diagram corresponds to a different value of the probabilities $\beta_{q}=\beta_{r}=\eta_{q}=\eta_{r}$ used for computing $\phi$

modified so that equality constraints of this type become part of the model.

\section{A constrained-BLIM model}

To be general, given the knowledge structure $(Q, \mathcal{K})$, two distinct equivalence relations $\sim_{\beta}$ and $\sim_{\eta}$ on the set $Q$ are defined such that, for $q, r \in Q$,

$q \sim_{\beta} r \quad \Longrightarrow \quad \beta_{q}=\beta_{r}$

and

$q \sim_{\eta} r \quad \Longrightarrow \quad \eta_{q}=\eta_{r}$

The equivalence relation $\sim_{\beta}$ imposes equality constraints to the probabilities $\beta_{q}$, whereas $\sim_{\eta}$ imposes equality constraints to the probabilities $\eta_{q}$. Denote with $[q]_{\beta}=\{r \in$ $\left.Q: q \sim_{\beta} r\right\}$ and with $[q]_{\eta}=\left\{r \in Q: q \sim_{\eta} r\right\}$ the equivalence classes of item $q \in Q$ induced by $\sim_{\beta}$ and $\sim_{\eta}$, respectively. Because of the equality constraints in Eqs. 4 and 5, the model comprises one careless error parameter for each of the classes $[q]_{\beta}, q \in Q$, and one lucky guess parameter for each of the classes $[q]_{\eta}, q \in Q$. The careless error parameters of the classes $[q]_{\beta}$ are denoted $\beta_{[q]}$, whereas those of the classes $[q]_{\eta}$ are denoted $\eta_{[q]}$. Hence, for $q \in Q, \beta_{r}=\beta_{[q]}$ for all $r \in[q]_{\beta}$ and $\eta_{r}=\eta_{[q]}$ for all $r \in[q]_{\eta}$. The number of free parameters of this model is $|\mathcal{K}|+\left|Q_{\beta}^{*}\right|+\left|Q_{\eta}^{*}\right|-1$, where $Q_{\beta}^{*}$ is the set of equivalence classes induced by $\sim_{\beta}$ and $Q_{\eta}^{*}$ is the set of equivalence classes induced by $\sim_{\eta}$.
For the specific purpose of the present article, namely testing item equivalence at the probabilistic level, this model is applied with the additional restriction that if $q$ and $r$ are equally informative items in $Q$, then both $\beta_{q}=\beta_{r}$ and $\eta_{q}=\eta_{r}$ must hold true.

The parameters of this model can be estimated by maximum likelihood via the expectation-maximization (EM) algorithm. The equations for updating the parameter estimates at each iteration of the algorithm are derived in Appendix A and in Appendix B, for complete data and for missing data, respectively.

It should be noted that the constrained BLIM (only for complete data) can be estimated through the R-package pks (Heller \& Wickelmaier, 2013). However, to the best of our knowledge, there are no references documenting the EM updating equations for this type of constrains.

\section{Simulation study}

Two different aims were at the basis of the simulation study. The former was to check the sensitivity of the likelihood ratio test in choosing the model that correctly specifies the instance equivalence. The latter was to study the effect of wrong assumptions about the instance equivalence on the parameter estimates of the models.

It has been decided to run simulations by using complete data. Therefore, the BLIM for complete data was used. 


\section{Simulation design and data set generation}

The BLIM and the constrained BLIM were used to generate the data sets by using two different knowledge structures $\mathcal{K}_{1}$ and $\mathcal{K}_{2}$. The former is equal to one of the two knowledge structures used in the empirical application (i.e., $\mathcal{K}_{D S}$ ). It assumes equal informativeness within each of twelve different pairs of instances. The latter structure used for generating the data sets, denoted by $\mathcal{K}_{2}$, assumes that the instances in one of the pairs specified in $\mathcal{K}_{1}$ are not equally informative. More details on the two structures follow.

For a knowledge domain $Q=\left\{q_{1}, q_{2}, \cdots, q_{24}\right\}$ of 24 items, the equivalence classes $q_{i}^{*}=\left\{q_{i}, q_{i+1}\right\}$ for all the odd numbers $i$ smaller than 24 were assumed in $\mathcal{K}_{1}$. The second knowledge structure $\left(Q, \mathcal{K}_{2}\right)$ was obtained by adding to $\mathcal{K}_{1}$ a certain number of states that violate the equal informativeness of the instances $q_{1}$ and $q_{2}$. Each of these new states contains one of the two instances but not the other. Thus, $\mathcal{K}_{1} \subset \mathcal{K}_{2}$ and, in $\mathcal{K}_{2}$, items $q_{1}$ and $q_{2}$ are not equally informative anymore. The resulting knowledge structure $\mathcal{K}_{2}$ contains 275 states, whereas $\mathcal{K}_{1}$ contains 165 states.

The local identifiability of the BLIM for both the knowledge structures $\mathcal{K}_{1}$ and $\mathcal{K}_{2}$ was tested through the BLIMIT function (Stefanutti et al., 2012) and no identifiability issues were found.

Three different scenarios were considered, in which the following three sets of assumptions concerning the two instances $q_{1}$ and $q_{2}$ were given:

- $q_{1}$ and $q_{2}$ are equally informative and their parameters are equal (the constrained BLIM with $\mathcal{K}_{1}$ was used for generating the data sets);

- $q_{1}$ and $q_{2}$ are equally informative but their parameters are not equal (the BLIM with $\mathcal{K}_{1}$ was used for generating the data sets);

- $q_{1}$ and $q_{2}$ are not equally informative and their parameters are not equal (the BLIM with $\mathcal{K}_{2}$ was used for generating the data sets).

The acronyms $\mathrm{cB} \mathcal{K}_{1}, \mathrm{~B} \mathcal{K}_{1}$ and $\mathrm{B} \mathcal{K}_{2}$ will be used to refer to these scenarios, respectively.

In order to study how the probability of detecting the true model is affected by the probabilities of the instances and by their error parameters, in each of the three scenarios a $6 \times 2$ factorial design was used. In particular, six different values of the probabilities $P\left(q_{1}\right)=\sum_{K \in \mathcal{K}_{q_{1}}} \pi_{K}$ and $P\left(q_{2}\right)=$ $\sum_{K \in \mathcal{K}_{q_{2}}} \pi_{K}$ and two different values of the parameters $\beta_{1}$ and $\beta_{2}$ were considered.

In order to manipulate the item probabilities, the following steps were carried out: (i) $P\left(q_{1}\right)$ was fixed to a specific value; (ii) A linear transformation was applied to states containing $q_{1}$ so that the sum of their probabilities was equal to $P\left(q_{1}\right)$; (iii) A second linear transformation was applied to the probabilities of the states not containing $q_{1}$ so that the sum of their probabilities was equal to $1-P\left(q_{1}\right)$; (iv) At this point, the probability of $q_{2}$ was computed by summing up the probabilities of all the states containing $q_{2}$. In this way the probability of $q_{1}$ was directly manipulated, whereas that of $q_{2}$ depended time to time upon the choice made for $q_{1}$. In the two scenarios $\mathrm{cB} \mathcal{K}_{1}$ and $\mathrm{B} \mathcal{K}_{1}$, the probabilities of the equally informative instances $q_{1}$ and $q_{2}$ were $P\left(q_{1}\right)=P\left(q_{2}\right) \in$ $\{.10, .25,40, .55, .70, .95\}$ (it is a property of both the BLIM and the constrained BLIM that equally informative items have also equal probabilities). In scenario $\mathrm{B} \mathcal{K}_{2}$, these two probabilities were $P\left(q_{1}\right) \in\{.10, .25,40, .55, .70, .95\}$ and $P\left(q_{2}\right) \in\{.34, .40, .45, .52, .59, .64\}$.

Concerning the two parameters $\beta_{1}$ and $\beta_{2}$, in scenario $\mathrm{cB} \mathcal{K}_{1} \beta_{1}=\beta_{2} \in\{.10, .40\}$, whereas in scenarios $\mathrm{B} \mathcal{K}_{1}$ and $\mathrm{B} \mathcal{K}_{2}, \beta_{1}=.10$ and $\beta_{2}=.40$ in the former manipulation, and $\beta_{1}=.20$ and $\beta_{2}=.30$ in the latter manipulation. This was done in order to manipulate the distance between the parameter values of the two instances (larger in the first manipulation). The simulation design is summarized in Table 1.

As regards the careless error of the other items of the domain (from 3 to 12) and the lucky guesses of all the 12 items, they were randomly drawn from a uniform distribution $U(0, .05)$ and kept constant across all the conditions. It was decided to keep the error rates of these items below .05 because higher values could have a negative effect on the reliability of the fit statistics (Stefanutti \& Robusto, 2009).

The probabilities of the knowledge states in each of the two structures $\mathcal{K}_{1}$ and $\mathcal{K}_{2}$ were randomly drawn from a uniform distribution $U(0,1)$ and then normalized to sum up to 1.

For each scenario and each of the $6 \times 2=12$ conditions, 100 samples were simulated by using two different sample sizes, that were 1000 or 286 . This last sample size was chosen because it is the same of the empirical application.

On the whole $3 \times 12 \times 2 \times 100=7,200$ data sets were generated.

\section{Methods}

The constrained BLIM estimated by using $\mathcal{K}_{1}$ and the BLIM estimated by using either $\mathcal{K}_{1}$ or $\mathcal{K}_{2}$ were applied to each generated data set. The parameters of the three models were estimated by maximum likelihood by using the EM algorithm (see Appendix A). A MATLAB function that implements the estimation procedures for both the BLIM and the constrained BLIM was developed by one of the authors (LS).

Three models stating three different assumptions concerning the instance equivalence were applied to the data 
Table 1 Values of $P\left(q_{1}\right), P\left(q_{2}\right), \beta_{1}$ and $\beta_{2}$ used for generating the data sets of the simulation study

\begin{tabular}{|c|c|c|c|c|c|c|c|c|c|c|c|c|}
\hline \multirow[b]{2}{*}{ Cond. } & \multicolumn{4}{|l|}{$\mathrm{cB} \mathcal{K}_{1}$} & \multicolumn{4}{|l|}{$\mathrm{B} \mathcal{K}_{1}$} & \multicolumn{4}{|l|}{$\mathrm{B} \mathcal{K}_{2}$} \\
\hline & $P\left(q_{1}\right)$ & $P\left(q_{2}\right)$ & $\beta_{1}$ & $\beta_{2}$ & $P\left(q_{1}\right)$ & $P\left(q_{2}\right)$ & $\beta_{1}$ & $\beta_{2}$ & $P\left(q_{1}\right)$ & $P\left(q_{2}\right)$ & $\beta_{1}$ & $\beta_{2}$ \\
\hline 1 & .10 & .10 & .1 & .1 & .10 & .10 & .1 & .4 & .10 & .34 & .1 & .4 \\
\hline 2 & .25 & .25 & .1 & .1 & .25 & .25 & .1 & .4 & .25 & .40 & .1 & .4 \\
\hline 3 & .40 & .40 & .1 & .1 & .40 & .40 & .1 & .4 & .40 & .45 & .1 & .4 \\
\hline 4 & .55 & .55 & .1 & .1 & .55 & .55 & .1 & .4 & .55 & .52 & .1 & .4 \\
\hline 5 & .70 & .70 & .1 & .1 & .70 & .70 & .1 & .4 & .70 & .59 & .1 & .4 \\
\hline 6 & .85 & .85 & .1 & .1 & .85 & .85 & .1 & .4 & .85 & .64 & .1 & .4 \\
\hline 7 & .10 & .10 & .4 & .4 & .10 & .10 & .2 & .3 & .10 & .34 & .2 & .3 \\
\hline 8 & .25 & .25 & .4 & .4 & .25 & .25 & .2 & .3 & .25 & .40 & .2 & .3 \\
\hline 9 & .40 & .40 & .4 & .4 & .40 & .40 & .2 & .3 & .40 & .45 & .2 & .3 \\
\hline 10 & .55 & .55 & .4 & .4 & .55 & .55 & .2 & .3 & .55 & .52 & .2 & .3 \\
\hline 11 & .70 & .70 & .4 & .4 & .70 & .70 & .2 & .3 & .70 & .59 & .2 & .3 \\
\hline 12 & .85 & .85 & .4 & .4 & .85 & .85 & .2 & .3 & .85 & .64 & .2 & .3 \\
\hline
\end{tabular}

sets, but just one was the model used for generating them. A hierarchy of three nested models was obtained: the BLIM based on $\mathcal{K}_{1}$ is nested into the BLIM based on $\mathcal{K}_{2}$ because $\mathcal{K}_{1} \subset \mathcal{K}_{2}$; the constrained BLIM based on $\mathcal{K}_{1}$ is nested into the BLIM based on $\mathcal{K}_{1}$ because it can be obtained from this last by imposing the equality constraints $\beta_{1}=\beta_{2}$ and $\eta_{1}=\eta_{2}$. It follows that the constrained BLIM based on $\mathcal{K}_{1}$ is also nested into the BLIM based on $\mathcal{K}_{2}$. The nested models were compared to each other by using the likelihood ratio (LR) test:

$L R=-2 \ln \frac{L_{M 0}}{L_{M 1}}$,

where $M 0$ and $M 1$ represent any two of the three models at issue, $L_{M 0}$ and $L_{M 1}$ are the maximized values of the likelihood function of the two models $M 0$ and $M 1$, and $M 1$ is the most complex model.

The proportion of data sets in which the LR test selected the most complex model was computed in all the 12 conditions of each of the three scenarios.

\section{Results}

The probability of selecting the correct model via the LR test did not vary with the instance probabilities. Differently from what we observed for the $\phi$-coefficient (see "A naïve method for testing instance equivalence"), the LR test seems not to be affected by the instance probabilities. For the sake of simplicity, in each of the three scenarios, the $6 \times 2$ conditions are reduced to two by taking averages across Conditions 1 to 6 and across Conditions 7 to 12 .

Figure 2 shows the most relevant results. In each panel of the figure, the sample size is on the $x$-axis and the proportions of cases in which the $p$-value of the LR test was less or equal to .05 is on the $y$-axis. Error bars indicate the $95 \%$ confidence intervals. Panel titles specify the two models being compared with the true model in parentheses.

The upper left panel displays the results concerning scenario $\mathrm{cB} \mathcal{K}_{1}$, when the true model was compared with the BLIM based on the true knowledge structure $\mathcal{K}_{1}$. It can be noted that: (i) when the error parameters of $q_{1}$ and $q_{2}$ are small (solid line in the figure), the proportion of cases in which the wrong (most complex) model is selected against the true model is .07 when $N=286$ and .05 when $N=1000$; (ii) when the error parameters of $q_{1}$ and $q_{2}$ are high (dashed line in the figure), these proportions slightly increased to .11 and .07 , respectively. Since these values are the proportions of samples incorrectly rejecting the true model, they can be interpreted as estimates of the type I error probability. These results suggest that the theoretical distribution of the Chi-square is reliable when the sample size is 1000 and the error parameters are quite low; whereas, with a simple size of 286 , this is not the case and a bootstrapped distribution is more appropriate for estimating the $p$-value of the LR test.

When the true model was compared with the BLIM estimated on the wrong structure $\mathcal{K}_{2}$, the correct model was always selected. This happened in all the conditions, irrespectively of the sample sizes.

In scenario $\mathrm{B} \mathcal{K}_{1}$ comparing the true model with the constrained BLIM estimated on $\mathcal{K}_{1}$ (upper right panel of Fig. 2), not surprisingly the model selection depended on the difference between the parameters $\beta_{1}$ and $\beta_{2}$. When the difference was large (solid line), the proportions of cases in which the correct model was selected were .94 when $N=286$, and 1.00 when $N=1000$. Whereas, when the difference between the error parameters was small (dashed line), the proportions of data sets in which the correct model 

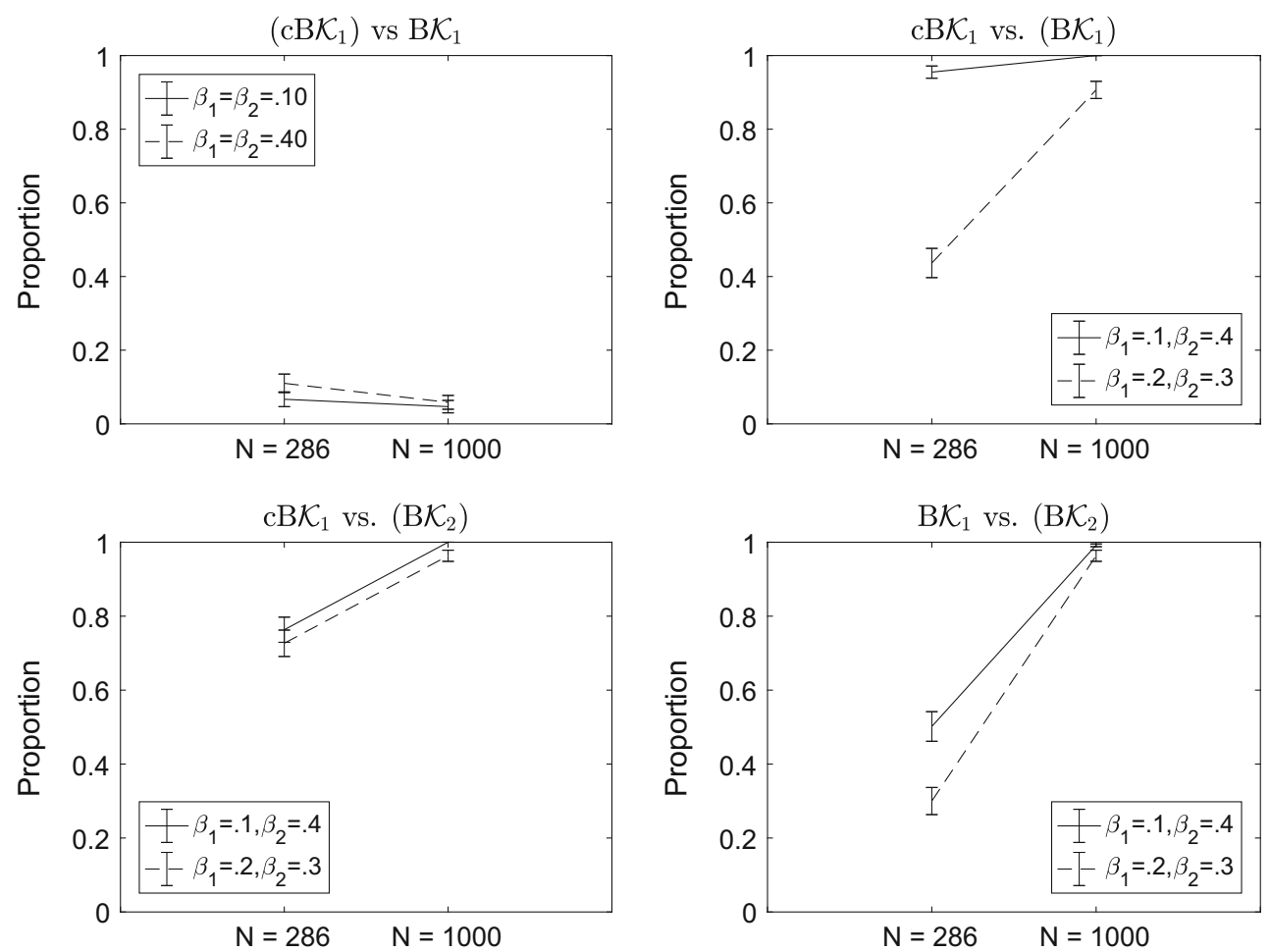

Fig. 2 Most relevant results of the simulation study. In each panel of the figure, the sample size is on the $x$-axis and the proportions of cases in which the $p$-value of the LR test was less or equal to .05 is on the $y$-axis. Error bars indicate the $95 \%$ confidence intervals. Panel titles specify the two models being compared with the true model in parentheses

was selected decreased to .44 when $N=286$, and to .91 when $N=1000$. The proportion of cases in which the BLIM is correctly selected against the constrained BLIM (that is the estimated probability of rejecting the false model when it is wrong) can be interpreted as the statistical power of the LR test. So that, the LR test is not sufficiently powerful when the sample size is as small as 286 and the error in the data is as large as .40. In the remaining conditions, the statistical power achieved satisfactory levels. Comparing the true model with the BLIM estimated by using the wrong structure $\mathcal{K}_{2}$, the LR test always selected the true model.

The left bottom panel of Fig. 2 displays the results obtained in scenario $\mathrm{B} \mathcal{K}_{2}$, when the true model was compared to the constrained BLIM estimated on $\mathcal{K}_{1}$. It can be noted that, when the sample size increases from 286 to 1000 , the proportion of cases in which the true model was selected increased from about .76 to about 1.00 . The difference between the two error parameter conditions was negligible. The right bottom panel displays the results obtained when the true model was compared to the BLIM estimated on $\mathcal{K}_{1}$. When sample size was 1000 , the power of the test was about .98 . When it was 286 , the power sharply decreased to about .40 . Not surprisingly, when the true model is the most complex one and the sample size is small, the LR test is not sufficiently powerful.

\section{Discussion}

On the whole, the results of the simulation study suggest that:

(i) when the instances of an item template are equivalent at both the deterministic and the probabilistic level, and the sample size is sufficiently high, the likelihood ratio test seems to be successful and reliable, irrespectively of the alternative model, of the amount of error in the data, and of the instance probabilities. Nevertheless, when the sample size is small, the bootstrap distribution, rather than the theoretical one, should be used for computing the $p$-value of the LR test;

(ii) when two instances are equivalent at the deterministic level but not at the probabilistic level and the true model is compared with a model assuming neither type of equivalence, the LR test is successful. Instead, it has to be used carefully when the true model is compared with a model assuming both types of equivalence. This is particularly true when the sample size is small and the error parameters are high. In fact, in this case, a small difference between the true error parameters could lead the LR test to fail because of its low power; 
(iii) the LR test seems very sensitive in detecting the cases in which the instances are equivalent neither at the deterministic nor at the probabilistic level, but only if the sample size is sufficiently large. Otherwise, the low power of the test could lead to a wrong decision in most cases.

\section{Empirical application}

The general purpose of the empirical application was to give an example of how a procedure based on the comparison between the BLIM and the constrained BLIM (illustrated in "Procedure and methods") can be applied for testing the equivalence among instances generated from the same "item template".

A collection of item templates has been developed by using the following procedure. Each item template was composed of variable and static components. The variable components were either qualitative (strings drawn from a set of words/sentences) or quantitative (numerical values belonging to specific intervals). When generating an instance, the values of the variable components were selected at random. There were 12 item templates for the topic "descriptive statistics" (DS), and 13 item templates for the topic "probability theory" (PT). As an example, Table 2 shows, for each of the two topics, three item templates and a pair of instances generated from each of them.

For both topics, the test was built by using exactly two instances for each item template. In this way, each student answered both instances. The test consisted of 50 instances: 24 for the DS topic, and 26 for the PT topic.

\section{Procedure and methods}

The 50 item instances were administered in two different sessions, one for each of the two topics, separated by a time interval of two weeks. In both sessions, the assessment lasted two hours. The test was administered to students via a computerized procedure. At the end of the assessment, an automatically generated report was provided to each student.

The response patterns with more than $50 \%$ of missing responses were removed from the data sets. Thus, the data sets used for the analysis were composed of 286 out of 361 students in the DS topic and of 344 out of 357 in the PT topic. The percentages of missing data observed in the final data sets were about 3\% (DS) and 2\% (PT).

For both topics, an iterative model comparison procedure for searching the model that best fitted the data was run. Figure 3 shows a diagram of the iterative procedure.

At the first step, the BLIM was fitted to the data with a knowledge structure $\mathcal{K}$ in which equal informativeness among the instances of the same item template was assumed. The structure $\mathcal{K}$ was obtained in two steps. First a temporary discriminative structure $\mathcal{K}^{*}$ was built on the item templates $q^{*}$. Then, each state $K^{*} \in \mathcal{K}^{*}$ was used as a guide for generating the states $K \in \mathcal{K}$. In particular, for any $q \in Q$

$q \in K \Longleftrightarrow q^{*} \in K^{*}$.

For the DS topic, $\mathcal{K}^{*}$ was constructed from a skill map (Doignon, 1994; Korossy, 1999; Ünlü et al., 2013) relating 12 skills to the 12 item templates (Table 3). For the PT topic, $\mathcal{K}^{*}$ was obtained from a skill map relating 9 skills to the 13 item templates (Table 4). The resulting structures $\mathcal{K}_{D S}$ and $\mathcal{K}_{P T}$, containing respectively 165 and 90 states, were obtained. Concerning the identifiability of the two structures $\mathcal{K}_{D S}$ and $\mathcal{K}_{P T}$, Spoto et al. (2013) have formulated a conjecture according to which, if $(Q, K)$ is the extension to equally informative items of a knowledge structure, then the BLIM is locally identifiable for $(Q, K)$. Furthermore, Heller (2017) refined the conjecture for subsets of the parameter space having positive Lebesgue measure. Anyway, an application of the BLIMIT function (Stefanutti et al., 2012) to $\mathcal{K}_{D S}$ and $\mathcal{K}_{P T}$ did not detect identifiability issues.

Coming back to the procedure, if the BLIM with $\mathcal{K}$ does not fit the data, it is possible that equal informativeness does not hold for some of the instances. Otherwise, at this stage, the conclusion that the instances of the item templates are equally informative can not be rejected and the procedure tests the parameter equality assumption. The constrained BLIM with the maximum number of equality constraints on the item parameters is fitted to the data. If the LR test selects the constrained BLIM against the BLIM, then the algorithm stops. Otherwise, a loop starts, in which equality constraints between the parameters of the instances are removed one at the time.

At each iteration of the loop, the equivalence class $q^{*}=$ $\{q, r\}$ for which the equality constraint is removed, is the one having the highest value of the parameter discrepancy

$\Delta_{q, r}=\frac{\left|\hat{\beta}_{q}-\hat{\beta}_{r}\right|}{\sqrt{\operatorname{var}\left(\hat{\beta}_{q}, \hat{\beta}_{r}\right)}}+\frac{\left|\hat{\eta}_{q}-\hat{\eta}_{r}\right|}{\sqrt{\operatorname{var}\left(\hat{\eta}_{q}, \hat{\eta}_{r}\right)}}$,

where: $\hat{\beta}_{q}$ (resp. $\hat{\eta}_{q}$ ) and $\hat{\beta}_{r}$ (resp. $\hat{\eta}_{r}$ ) are the estimates of the careless error (rs. lucky guess) parameters of the two instances $q$ and $r ; \operatorname{var}\left(\hat{\beta}_{q}, \hat{\beta}_{r}\right)$ and $\operatorname{var}\left(\hat{\eta}_{q}, \hat{\eta}_{r}\right)$ are the variances of the differences between the parameter estimates of the two instances $q$ and $r$, when these parameter estimates are regarded as non-independent random variables.

The goodness of fit of the models was tested by using the LR test. A parametric bootstrap procedure (Efron, 1979) over 500 replications was run for computing the $p$-value of the LR test. This was necessary because the 


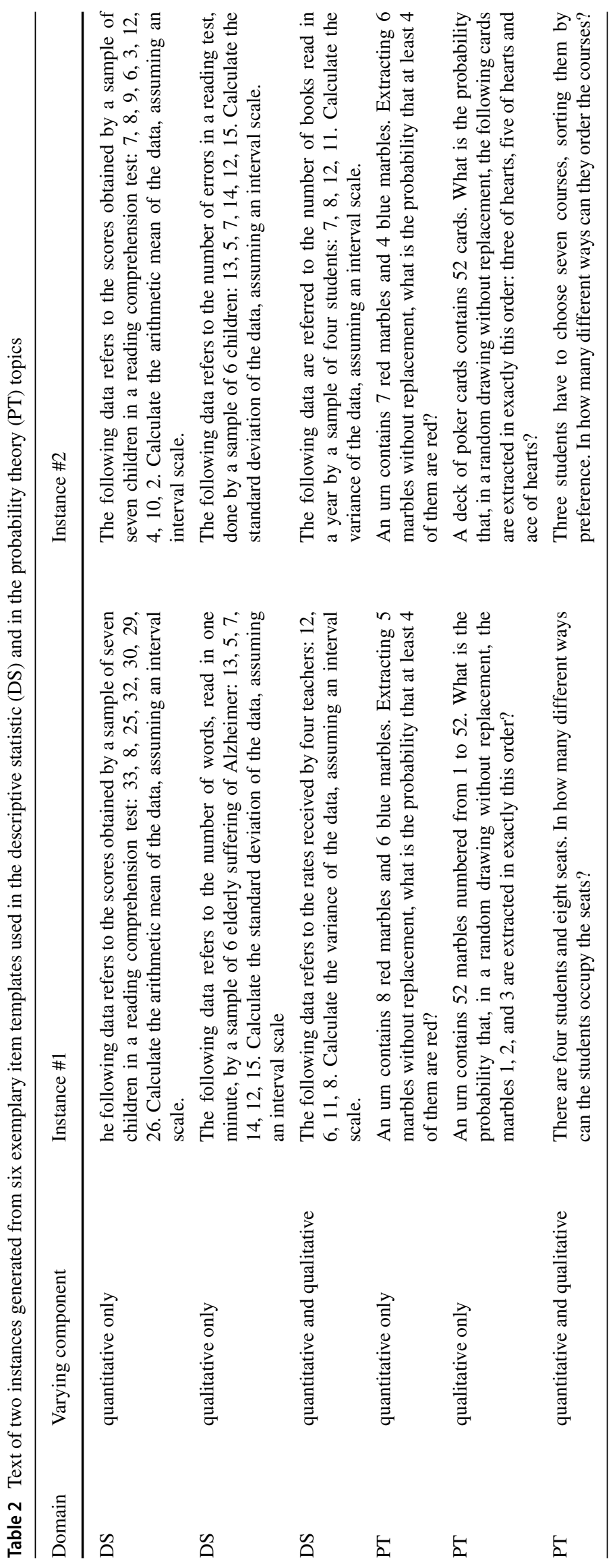




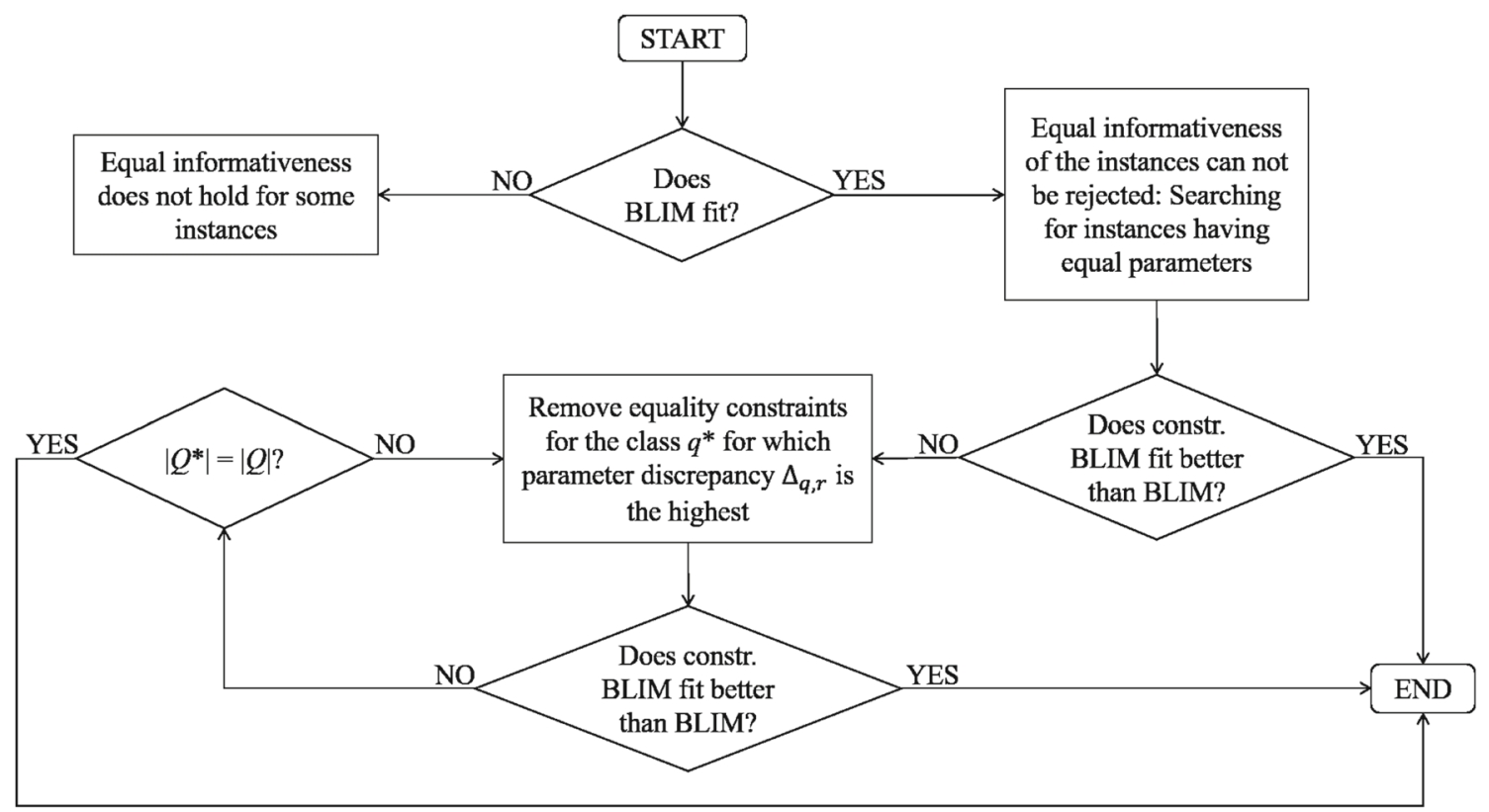

Fig. 3 Iterative model comparison procedure used in the empirical application for searching the model that best fitted the data. See text for a detailed explanation

approximation to the asymptotic distribution of the Chisquare statistic lacks accuracy for large and sparse data matrices and this was the case of the present empirical application $\left(2^{12}=4096\right.$ theoretical response patterns over 269 observed response patterns for the DS topic and $2^{13}=$ 8192 theoretical response patterns over 338 theoretical ones for the PT topic). For each of the two empirical data sets, the parametric bootstrap was performed in the following way. The parameter estimates obtained by fitting the model to the data were used to generate 500 data sets of the same size of the observed samples $\left(N_{D S}=286\right.$ and $\left.N_{P T}=344\right)$. Then,

Table 3 The skill map used for generating the structure $\mathcal{K}_{D S}$ of the empirical application

\begin{tabular}{llll}
\hline Template $q^{*}$ & Skill $s$ & Template $q^{*}$ & Skill $s$ \\
\hline 1 & $\{d\}$ & 7 & $\{c, k\}$ \\
2 & $\{a, d, i, j, l\}$ & 8 & $\{b, c, k\}$ \\
3 & $\{d, i, j, l\}$ & 9 & $\{f, g, h\}$ \\
4 & $\{d, e, g\}$ & 10 & $\{f, g\}$ \\
5 & $\{c\}$ & 11 & $\{d, j\}$ \\
6 & $\{k\}$ & 12 & $\{d, i, j\}$ \\
\hline
\end{tabular}

Columns 1 and 3 list the 12 item templates $q^{*}$, whereas columns 2 and 4 list the set of relevant skills

(a) Calculate the standard deviation; (b) Solve exercises that require $z$-scores; (c) Invert a formula; (d) Calculate a mean; (e) Calculate the mean for grouped data; (f) Calculate the $n$-th percentile; (g) Calculate a proportion; (h) Calculate a percentile rank; (i) Calculate the squared deviation from the mean; (j) Calculate the deviation from the mean; (k) Apply $z$-scores to practical cases; (l) Calculate the variance the model was fitted to each of the 500 simulated data sets, and the proportion of cases in which the LR test was greater than that obtained for the empirical data set was computed.

\section{Results}

Concerning the DS topic, at the first step of the iterative model comparison procedure, the BLIM estimated with the knowledge structure $\mathcal{K}_{D S}$ obtained a bootstrapped $p$-value

Table 4 The skill map used for generating the structure $\mathcal{K}_{P T}$ of the empirical application

\begin{tabular}{llll}
\hline Template $q^{*}$ & Skill $s$ & Template $q^{*}$ & Skill $s$ \\
\hline 1 & $\{b\}$ & 8 & $\{a, b\}$ \\
2 & $\{b, i\}$ & 9 & $\{f\}$ \\
3 & $\{e\}$ & 10 & $\{a, b, i\}$ \\
4 & $\{b, d, e\}$ & 11 & $\{f, g\}$ \\
5 & $\{b, c\}$, & 12 & $\{b, f, g\}$ \\
6 & $\{b, c, d, e\}$ & 13 & $\{b, f, g, h\}$ \\
7 & $\{b, c, i\}$ & & \\
\hline
\end{tabular}

Columns 1 and 3 list the 13 item templates $q^{*}$, whereas columns 2 and 4 list the set of relevant skills

(a) Application of Bernoulli rule; (b) Application of combinations;

(c) Solving problems requiring the repeated use of combinations;

(d) Solving problems requiring the use of both $k$-permutations and combinations; (e) Solving problems by using $k$-permutations; (f) Computing the probability of an event in a uniform probability space; (g) Applying the chain rule of probabilities; (h) Computing the cumulative probability, or its complement, in sample draws; (i) Sums of repeated applications of combinations 
of .07. Unfortunately, this value cannot lead to a conclusive result about the equivalence of the instances. Nevertheless, as an example, the procedure run in the case of a non rejection of the BLIM is presented.

Figure 4 shows the careless error (top panel) and the lucky guess (bottom panel) parameter estimates obtained by the BLIM for the two instances of the 12 item templates. In the figure, the 12 item templates are displayed on $x$ axis whereas the parameter probabilities are displayed on the $y$-axis. A circle denotes the first instance of the item template, whereas a star denotes the second instance. Error bars indicate $95 \%$ bootstrapped confidence intervals of the estimates.

The average values of the parameter estimates were quite low $\left(\bar{\beta}_{q}=.16\right.$ and $\left.\bar{\eta}_{q}=.16\right)$ and they also had small confidence intervals. The largest error parameter estimates were observed for the careless error of two instances of Item template 9: Their values did not exceed .36 and .42. Most of the parameter estimates of the two instances of the 12 item templates did not differ significantly. The only exceptions were the lucky guesses of Items 1 and 12 and the careless errors of Item 10.

At the second step of the iterative procedure, the constrained BLIM was estimated assuming parameter equality constrains for all the pairs of equivalent instances
(12 equivalence classes, each containing two instances). From the comparison of this model against the BLIM, the bootstrapped $p$-value of the LR test was equal to .004 . With a type I error probability of .05 , the obtained $p$ value was not significant, meaning that the most complex model (BLIM) was selected. As expected, the equivalence assumption of the error parameters between the two instances of all the 12 item templates was too strong.

At this point, the iterative procedure started the loop in which equality constrains on the instance parameters were removed one at the time. The procedure terminated after two iterations, leading to a constrained BLIM in which only two equality constrains were removed: The constraint between the error parameters of the two instances of Item template 1 and the constraint between those of the two instances of the Item template 10. Comparing this model with the BLIM, a bootstrapped $p$-value of .16 was obtained for the LR statistic. In light of what observed in the simulation study (see right top panel of Fig. 2), the test was high powered $\left(N=286, \eta_{1}^{*} \simeq\{.1, .4\}\right.$ and $\left.\beta_{10}^{*} \simeq\{.1, .3\}\right)$.

Summarizing, the results of the iterative procedure suggest that the two instances generated from each of the item templates of the DS topic are equally informative. Moreover, 10 out of 12 item templates generated instances with equal careless error and lucky guess parameters. As
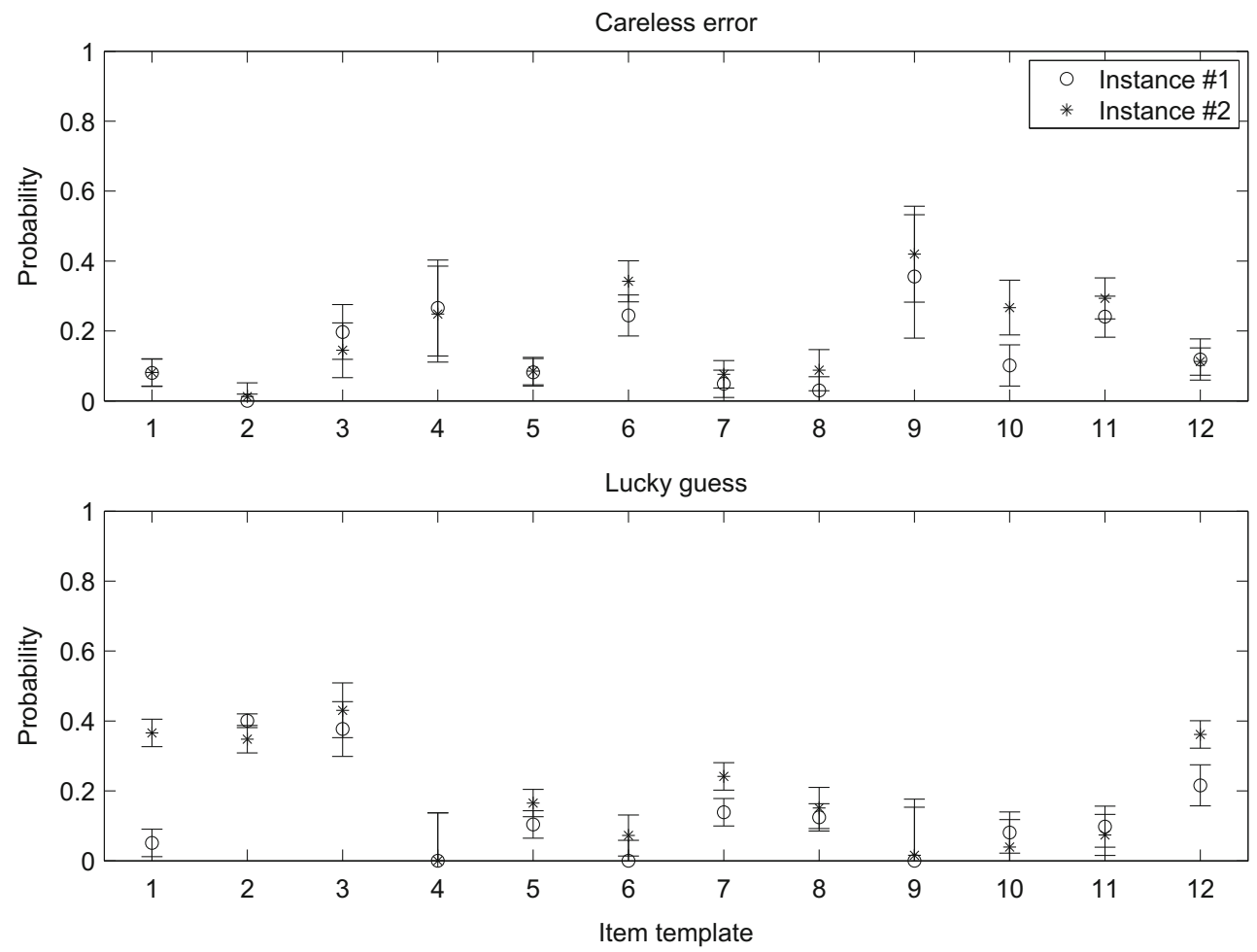

Fig. 4 Careless error (top panel) and lucky guess (bottom panel) parameter estimates of the BLIM for the two instances of the 12 item templates in the "descriptive statistic" topic. The 12 item templates are displayed on the $x$-axis, whereas the parameter probability is on the $y$-axis. A circle denotes the first instance of an item template, whereas a star denotes the second instance. Error bars indicate $95 \%$ confidence intervals of the estimates 

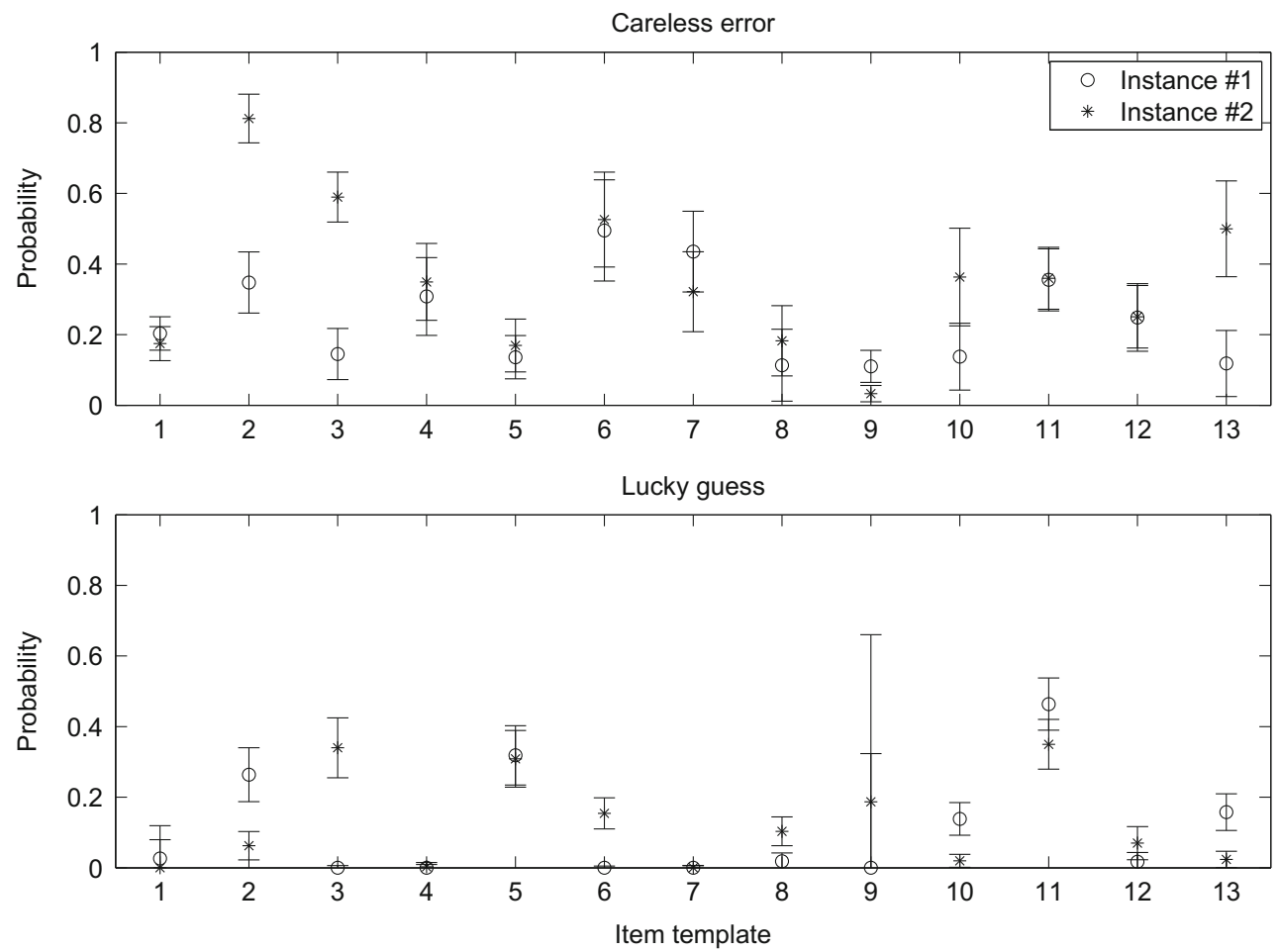

Fig. 5 Careless error (top panel) and lucky guess (bottom panel) parameter estimates of the BLIM for the two instances of the 13 item templates in the "probability theory" topic. The 13 item templates are displayed on the $x$-axis, whereas the parameter probability is on the $y$-axis. A circle denotes the first instance of an item template, whereas a star denotes the second instance. Error bars indicate $95 \%$ confidence intervals of the estimates

it will be discussed below, for the two remaining item templates the varying component was quantitative only.

The same iterative procedure was applied to the data set concerning the PT topic. At the first step of the procedure, the BLIM estimated with $\mathcal{K}_{P T}$ obtained a bootstrapped $p$ value of .10. Also in this topic, a conclusive decision about the model fit can not be taken. Nevertheless, as an example, the procedure run in the case of a non rejection of the BLIM is presented.

Figure 5 shows the careless error (top panel) and the lucky guess (bottom panel) parameter estimates obtained by the BLIM for the two instances of the 13 item templates. The figure reads as Fig. 4.

It is clear from the figure that the results obtained in this topic were more articulated than those found in the previous one, for the following reasons: (i) on average, the estimates of the careless errors were high $\left(\bar{\beta}_{q}=.30\right)$, whereas those of the lucky guesses were low ( $\bar{\eta}_{q}=.12$ ); (ii) some parameter estimates were very high (see, e.g., instances 2, 3, 6, and 13), indicating a possible misspecification of some knowledge states belonging to $\mathcal{K}_{P T}$; (iii) there are 5 item templates out of 13 (i.e., numbers $2,3,6,10$, and 13) whose instances had very different parameter estimates; and (iv) confidence intervals of the parameter estimates of some instances were large, in particular those of Item template 9.
So that, it was not a surprise to observe that, at the second step of the iterative procedure, the constrained BLIM did not fit the data. Furthermore, the algorithm removing equality constraints took 6 loops. This means that the error parameters of the instances generated by 6 different item templates (i.e., numbers 2, 3, 6, 9, 10, and 13) were not equal to one another. As discussed below, two different varying components characterized these items.

\section{Discussion}

The reasons for the nonequivalence among the instances of some item templates should be sought in their text. In the case of the DS topic, the varying component of both the item templates generating non equivalent instances (1 and 10) was quantitative only. The two instances of Item template 1 are displayed in the first row of Table 2. Comparing the two instances, it can be noted that the values used in Instance \#1 were systematically higher than those of Instance \#2. It is possible that this difference made Instance \#1 more difficult than Instance \#2. Since in our model the two instances were equally informative, any substantial difference between the two can only be accounted by their careless error or luckyguess parameter estimates. More specifically, this situation could result in either a large careless error for Instance \#1, 
or a large lucky guess for Instance \#2, or both. In our case, a large lucky guess for Instance \#2 was observed. For the two instances of Item template 10, similar considerations apply, but the case of a large careless error for Instance \#2 was observed.

In the PT topic, six different item templates generated nonequivalent instances. The variable components of Items 2,3 , and 6 were quantitative and qualitative, whereas those of Items 9, 10, and 13 were quantitative only.

To give an example of an item from the former group, consider the two instances generated by the Item template 2 . The qualitative varying component was of a linguistic type. In particular, in the first instance the expression "five or more" was used, whereas in the second one the expression "at least four" was used. Despite the two expressions have the same meaning, the first one is more explicit than the other. Looking at the item parameter estimates (Fig. 5), both the careless error and the lucky guess of the two instances differ one another. In particular, the careless error of Instance \#2 is higher than that of Instance \#1, whereas the lucky guess of Instance \#2 is lower than that of Instance \#1. These results suggest that the expression "at least" could lead to a more difficult instance than the expression "more than".

Concerning Item templates 9, 10, and 13, the quantitative variation component determined the number of different calculations required for solving the problem. To give an example, consider Item template 13, whose instances are displayed in the fourth row of Table 2. The number of computations is lower in Instance \#1 ("at least 4 red marbles out of 5 extractions") than in Instance \#2 ("at least 4 red marbles out of 6 extractions" ). It is likely that this difference gives rise to a higher careless error for Instance \#2 than for Instance \#1, and to a lower lucky guess for Instance \#2 than for Instance \#1. Similar considerations can be done for Item template 10. Concerning Item template 9 , the high variance does not allow any conclusion about instance parameters equality.

A final interesting consideration regards the PT topic. For the two Item templates 11 and 12 belonging to the qualitative only variable component, the context is very different from one instance to the other. The two instances of Item template 11 are displayed in the fifth row of Table 2. As it can be seen, the former instance refers to "urns and marbles", whereas the other one to "playing cards" (concerning Item template 12, one instance refers to the "human beings" and the other to the "urns and marbles"). Nevertheless, the error parameters of the two instances of both item templates were found to be equivalent. This result is not unexpected given that exercises from the considered contexts were very common in classroom.

\section{Conclusions}

Since the use of computerized test for knowledge assessment increased very fast in the last years, developing methods for automatic item generation imposes itself. This is because the items are repeatedly administered, with a detrimental effect on their usefulness for the assessment. Assuming that a particular method for developing a certain number of instances from the same item template exists, the question of how the equivalence among different instances may be tested remains open. This is a very important aspect because two students answering to different instances of the same item template have to be assessed in the same way, but also because the same student answering to different instances of the same item template should answer (excluding careless errors or lucky guesses) in the same way.

In the present study, the question raised above was addressed within the knowledge space theory framework (KST; Albert \& Lukas 1999; Doignon \& Falmagne 1999; Falmagne \& Doignon 2011). Two different ways of considering the equivalence among instances have been proposed: The former is at a deterministic level and requires that all the instances of an item template must belong to exactly the same knowledge states; The latter adds a probabilistic level to the deterministic one, by introducing equality constraint assumptions regarding the error parameters of the instances. The former type of equivalence is modeled by using the BLIM (Falmagne \& Doignon 1988a, b) with a knowledge structure in which the instances of the same item template belong to exactly the same states of the structure. The latter is modeled by a constrained BLIM in which the careless error and the lucky-guess parameters are equal among all the instances generated by the same item template. If both the models are fitted to the same data set, a likelihood ratio test can be applied for selecting the one that best fits the data. The two models were applied in both a simulation study and an empirical application.

The results of the simulation study showed that the LR test can be successfully applied when: (i) the instances of an item template are equivalent at both the deterministic and the probabilistic level; and (ii) the instances are neither equivalent at the deterministic nor at the probabilistic level.

In the empirical application, the text of some item templates was produced by using two different types of variable components: a "qualitative" component and a "quantitative" component. The aim was to study their effect on the equivalence of the instances generated from item templates in the "descriptive statistics" and "probability theory" topics. 
Concerning the equivalence at the probabilistic level, the most part of the item templates generated instances with equal parameters in both topics (10 out of 12 for the DS topic and 7 out of 13 for the PT topic). For the nonequivalent item templates, the variable components were "quantitative only" or "quantitative and qualitative". The "quantitative" component produced instances having different error parameters when: (i) the values were selected from different numerical intervals; or (ii) the values determined a different number of computations involved in the solution of the problem. As regards the "qualitative" component, it seems that different text wording (e.g., "at least", "more than") generated instances with different error parameters.

Further studies are needed for examining the effect of other types of "qualitative variation" (e.g., the "syntactic variation") on the instance equivalence. Moreover, it would be interesting to study the effect of the student's knowledge of the context (e.g., "urns" and "cards"). In our research, the choice of one or the other context generated equivalent instances because the students were exposed to both types of problems in classroom.

In both the simulation study and the empirical application, the equivalence of exactly two instances generated by the same item template was tested. However, it is worth noting that the proposed procedure is suitable for any number of equivalent instances. Further studies could investigate situations with different number of instances generated from the same item template.

Acknowledgements The research developed in this article was carried out under the research project CPDR152105 "Learning how students learn. Mathematical modeling of learning processes in intelligent tutoring system navigation", funded by the University of Padua, Italy.

\section{Appendix A: Equations for the parameter estimation of the constrained BLIM}

The parameters of the constrained BLIM can be estimated by maximum likelihood, under the equality constraints specified in "Modeling instance equivalence in KST", through the EM algorithm (Stefanutti and Robusto, 2009). Let $(\mathcal{R}, F)$, with $\mathcal{R}=2^{Q}$ and $F: \mathcal{R} \rightarrow \mathbb{R}$, denote a sample of size $N=\sum_{R \in \mathcal{R}} F(R)$. For $R \in \mathcal{R}, F(R) \geq 0$ is the observed frequency of $R$ in the sample. Given the parameter vector $\theta=(\beta, \eta, \pi)$, the conditional expectation of the constrained BLIM's complete log-likelihood equals that of the BLIM (see, e.g., Stefanutti \& Robusto, 2009):

$$
E(\ln L \mid \mathcal{R}, F, \theta)=\sum_{R \in \mathcal{R}} \sum_{K \in \mathcal{K}} F(R) P_{\theta}(K \mid R) \ln P(R \mid K) \pi_{K},
$$

where, for $K \in \mathcal{K}$ and $R \in \mathcal{R}$,

$$
P_{\theta}(K \mid R)=\frac{P(R \mid K) \pi_{K}}{\sum_{L \in \mathcal{K}} P(R \mid L) \pi_{L}}
$$

is the posterior probability of knowledge state $K$, given response pattern $R$ and the model parameters $\theta$. The expected log-likelihood (8) turns out to be the sum of the two terms

$\mathcal{E}_{1}=\sum_{R \in \mathcal{R}} \sum_{K \in \mathcal{K}} F(R) P_{\theta}(K \mid R) \ln P(R \mid K)$,

and

$\mathcal{E}_{2}=\sum_{R \in \mathcal{R}} \sum_{K \in \mathcal{K}} F(R) P_{\theta}(K \mid R) \ln \pi_{K}$.

It can be easily seen that, in the BLIM, $\mathcal{E}_{1}$ only depends on the $\beta_{q}$ and $\eta_{q}$ parameters, whereas $\mathcal{E}_{2}$ only depends on the $\pi_{K}$ probabilities. Since in the constrained BLIM the $\beta_{q}$ and $\eta_{q}$ item parameters are equal to the $\beta_{[q]}$ and $\eta_{[q]}$ class parameters respectively, it turns out that in the constrained BLIM $\mathcal{E}_{1}$ only depends on the $\beta_{[q]}$ and $\eta_{[q]}$ item class parameters. The term $\mathcal{E}_{1}$ is maximized by setting to zero its partial derivatives with respect to the $\beta_{[q]}$ and $\eta_{[q]}$ parameters of the item classes. The first partial derivative with respect to $\beta_{[q]}, q \in Q$ is

$$
\frac{\partial \mathcal{E}_{1}}{\partial \beta_{[q]}}=\sum_{R \in \mathcal{R}} \sum_{K \in \mathcal{K}} F(R) P_{\theta}(K \mid R) \frac{\partial \ln P(R \mid K)}{\partial \beta_{[q]}}
$$

where

$$
\begin{aligned}
\frac{\partial \ln P(R \mid K)}{\partial \beta_{[q]}} & =\sum_{r \in K \backslash R} \frac{\partial \ln \beta_{r}}{\partial \beta_{[q]}}+\sum_{r \in K \cap R} \frac{\partial \ln \left(1-\beta_{r}\right)}{\partial \beta_{[q]}} \\
& =\sum_{r \in K \backslash R} \frac{1}{\beta_{r}} \frac{\partial \beta_{r}}{\partial \beta_{[q]}}-\sum_{r \in K \cap R} \frac{1}{1-\beta_{r}} \frac{\partial \beta_{r}}{\partial \beta_{[q]}} .
\end{aligned}
$$

But

$$
\frac{\partial \beta_{r}}{\partial \beta_{[q]}}=\left\{\begin{array}{l}
1 \text { if } r \in[q]_{\beta} \\
0 \text { if } r \notin[q]_{\beta},
\end{array}\right.
$$

hence

$\frac{\partial \ln P(R \mid K)}{\partial \beta_{[q]}}=\frac{\left|[q]_{\beta} \cap K \backslash R\right|}{\beta_{[q]}}-\frac{\left|[q]_{\beta} \cap K \cap R\right|}{1-\beta_{[q]}}$.

Thus we have:

$\frac{\partial \mathcal{E}_{1}}{\partial \beta_{q}}=\sum_{R \in \mathcal{R}} \sum_{K \in \mathcal{K}} F(R) P_{\theta}(K \mid R)\left(\frac{\left|[q]_{\beta} \cap K \backslash R\right|}{\beta_{[q]}}-\frac{\left|[q]_{\beta} \cap K \cap R\right|}{1-\beta_{[q]}}\right)$.

Setting to zero the right-hand side of this equation and solving for $\beta_{[q]}$ yields

$\beta_{[q]}=\frac{\sum_{K \in \mathcal{K}} \sum_{R \in \mathcal{R}}\left|[q]_{\beta} \cap K \backslash R\right| F(R) P_{\theta}(K \mid R)}{\sum_{K \in \mathcal{K}} \sum_{R \in \mathcal{R}}\left|[q]_{\beta} \cap K\right| F(R) P_{\theta}(K \mid R)}$. 
A similar development for $\eta_{[q]}$ yields

$\eta_{[q]}=\frac{\sum_{K \in \mathcal{K}} \sum_{R \in \mathcal{R}}\left|[q]_{\eta} \cap R \backslash K\right| F(R) P_{\theta}(K \mid R)}{\sum_{K \in \mathcal{K}} \sum_{R \in \mathcal{R}}\left|[q]_{\eta} \backslash K\right| F(R) P_{\theta}(K \mid R)}$.

\section{Appendix B: Estimation and fit of the constrained BLIM for MAR data}

For deriving the updating equations of the EM for the case of missing-at-random (MAR) data, we draw upon a recent extension of the BLIM to MAR data derived by de Chiusole et al. (2015a) and further developed by Anselmi et al. (2016). A response pattern where some of the responses are missing is represented by a partition $(R, M, W)$ of $Q$. The set $R$ (respectively $W$ ) contains all items that received a correct (resp., wrong) answer, whereas $M$ is the collection of all items that received no answer. If the data are missing at random (MAR, Rubin 1976) then the following equation extends the BLIM to missing data: given any response pattern $(R, M, W)$ and any knowledge state $K \in \mathcal{K}$,

$P(R, M, W \mid K)=\left(\prod_{q \in W \cap K} \beta_{q}\right)\left(\prod_{q \in R \cap K}\left(1-\beta_{q}\right)\right)\left(\prod_{q \in W \backslash K}\left(1-\eta_{q}\right)\right)\left(\prod_{q \in R \backslash K} \eta_{q}\right)$.

Let If be the collection of all partitions $(R, M, W)$ of $Q$. A sample is represented by the pair (II, $F$ ), with $F: \mathbb{I} \rightarrow \mathbb{R}$ such that $F(R, W, M) \geq 0$ and $\sum_{(R, W, M) \in \mathbb{I}} F(R, W, M)=N$ is the sample size.
The conditional expectation of the model's complete log-likelihood is:

$E(\ln L \mid \mathbb{I}, F, \theta)=\sum_{(R, M, W) \in \mathbb{I}} \sum_{K \in \mathcal{K}} F(R, M, W) P_{\theta}(K \mid R, M, W) \ln P(R, M, W \mid K) \pi_{K}$,

where, for $K \in \mathcal{K}$ and $(R, M, W) \in \mathbb{I}$,

$P_{\theta}(K \mid R, M, W)=\frac{P(R, M, W \mid K) \pi_{K}}{\sum_{L \in \mathcal{K}} P(R, M, W \mid L) \pi_{L}}$

is the posterior probability of $K$, given $(R, M, W)$ and $\theta$. The expected log-likelihood (9) turns out to be the sum of the two terms

$\mathcal{E}_{1}^{*}=\sum_{(R, M, W) \in \mathbb{I}[} \sum_{K \in \mathcal{K}} F(R, M, W) P_{\theta}(K \mid R, M, W) \ln P(R, M, W \mid K)$,

and

$\mathcal{E}_{2}^{*}=\sum_{R, M, W \in \mathbb{I}} \sum_{K \in \mathcal{K}} F(R, M, W) P_{\theta}(K \mid R, M, W) \ln \pi_{K}$.

As in the case of the BLIM with complete data, $\mathcal{E}_{1}^{*}$ only depends on the $\beta_{q}$ and $\eta_{q}$ parameters, whereas $\mathcal{E}_{2}^{*}$ only depends on the $\pi_{K}$ probabilities.

For estimating the constrained BLIM with MAR data, the term $\mathcal{E}_{1}^{*}$ is maximized by setting to zero its partial derivatives with respect to the $\beta_{[q]}$ and $\eta_{[q]}$ parameters of the item classes. The first partial derivative with respect to $\beta_{[q]}, q \in Q$ is

$\frac{\partial \mathcal{E}_{1}^{*}}{\partial \beta_{[q]}}=\sum_{(R, M, W) \in \mathbb{I}} \sum_{K \in \mathcal{K}} F(R, M, W) P_{\theta}(K \mid R, M, W) \frac{\partial \ln P(R, M, W \mid K)}{\partial \beta_{[q]}}$

where

$$
\begin{aligned}
\frac{\partial \ln P(R, M, W \mid K)}{\partial \beta_{[q]}} & =\sum_{r \in W \cap K} \frac{\partial \ln \beta_{r}}{\partial \beta_{[q]}}+\sum_{r \in R \cap K} \frac{\partial \ln \left(1-\beta_{r}\right)}{\partial \beta_{[q]}} \\
& =\sum_{r \in W \cap K} \frac{1}{\beta_{r}} \frac{\partial \beta_{r}}{\partial \beta_{[q]}}-\sum_{r \in R \cap K} \frac{1}{1-\beta_{r}} \frac{\beta_{r}}{\beta_{[q]}} .
\end{aligned}
$$

But, again,

$\frac{\partial \beta_{r}}{\partial \beta_{[q]}}=\left\{\begin{array}{l}1 \text { if } r \in[q]_{\beta} \\ 0 \text { if } r \notin[q]_{\beta}\end{array}\right.$

hence

$\frac{\partial \ln P(R, M, W \mid K)}{\partial \beta_{[q]}}=\frac{\left|[q]_{\beta} \cap W \cap K\right|}{\beta_{[q]}}-\frac{\left|[q]_{\beta} \cap R \cap K\right|}{1-\beta_{[q]}}$.

Thus we have:

$\frac{\partial \mathcal{E}_{1}}{\partial \beta_{[q]}}=\sum_{(R, M, W) \in \mathcal{R}} \sum_{K \in \mathcal{K}} F(R, M, W) P_{\theta}(K \mid R, M, W)\left(\frac{\left|[q]_{\beta} \cap W \cap K\right|}{\beta_{[q]}}-\frac{\left|[q]_{\beta} \cap R \cap K\right|}{1-\beta_{[q]}}\right)$. 
Setting the right-hand term of this equation equal to zero and solving for $\beta_{[q]}$,

$\beta_{[q]}=\frac{\sum_{(R, M, W) \in \mathbb{I}} \sum_{K \in \mathcal{K}} F(R, M, W) P_{\theta}(K \mid R, M, W)\left|[q]_{\beta} \cap W \cap K\right|}{\sum_{(R, M, W) \in \mathbb{I}} \sum_{K \in \mathcal{K}} F(R, M, W) P_{\theta}(K \mid R, M, W)\left(\left|[q]_{\beta} \cap W \cap K\right|+\left|[q]_{\beta} \cap R \cap K\right|\right)}$.

But $R \cap W=\emptyset$, hence $\left([q]_{\beta} \cap W \cap K\right) \cap\left([q]_{\beta} \cap R \cap K\right)=$ $\emptyset$, therefore

$$
\begin{aligned}
\left|[q]_{\beta} \cap W \cap K\right|+\left|[q]_{\beta} \cap R \cap K\right| & =\left|\left([q]_{\beta} \cap W \cap K\right) \cup\left([q]_{\beta} \cap R \cap K\right)\right| \\
& =\left|\left([q]_{\beta} \cap K\right) \cap(R \cup W)\right| \\
& =\left|\left([q]_{\beta} \cap K\right) \cap(Q \backslash M)\right| \\
& =\left|\left([q]_{\beta} \cap K \backslash M\right)\right| .
\end{aligned}
$$

Hence we obtain:

$\beta_{[q]}=\frac{\sum_{(R, M, W) \in \mathbb{I}} \sum_{K \in \mathcal{K}} F(R, M, W) P_{\theta}(K \mid R, M, W)\left|[q]_{\beta} \cap W \cap K\right|}{\sum_{(R, M, W) \in \mathbb{I}} \sum_{K \in \mathcal{K}} F(R, M, W) P_{\theta}(K \mid R, M, W)\left|[q]_{\beta} \cap K \backslash M\right|}$.

A similar development for $\eta_{[q]}$ yields

$\eta_{[q]}=\frac{\sum_{(R, M, W) \in \mathbb{I}} \sum_{K \in \mathcal{K}} F(R, M, W) P_{\theta}(K \mid R, M, W)\left|[q]_{\eta} \cap R \backslash K\right|}{\sum_{(R, M, W) \in \mathbb{I}} \sum_{K \in \mathcal{K}} F(R, M, W) P_{\theta}(K \mid R, M, W)\left|[q]_{\eta} \backslash(M \cup K)\right|}$.

\section{References}

Albert, D., \& Lukas, J. (1999). Knowledge spaces: Theories, empirical research, and applications. Mahwah, New Jersey, London: Lawrence Erlbaum Associates.

Anselmi, P., Robusto, E., Stefanutti, L., \& de Chiusole, D. (2016). An upgrading procedure for adaptive assessment of knowledge. Psychometrika, 81(2), 461-482.

Anselmi, P., Stefanutti, L., de Chiusole, D., \& Robusto, E. (2017). The assessment of knowledge and learning in competence spaces: The gain-loss model for dependent skills. British Journal of Mathematical and Statistical Psychology, 70(3), 457479.

Arendasy, M. E., \& Sommer, M. (2012). Using automatic item generation to meet the increasing item demands of high-stakes educational and occupational assessment. Learning and individual differences, 22(1), 112-117.

Bolt, D. (2007). The present and future of IRT-based cognitive diagnostic models (ICDMs) and related methods. Journal of Educational Measurement, 44(4), 377-383.

Bormuth, J. (1969). On a theory of achievement test items. Chicago: University of Chicago Press.
Cosyn, E., Doble, C., Falmagne, J. C., Lenoble, A., Thiéry, N., \& Uzun, H. (2013). Assessing mathematical knowledge in a learning space. Knowledge Spaces (pp. 27-50). Berlin: Springer.

Cui, Y., Gierl, M. J., \& Chang, H. H. (2012). Estimating classification consistency and accuracy for cognitive diagnostic assessment. Journal of Educational Measurement, 49(1), 19-38.

de Chiusole, D., Anselmi, P., Stefanutti, L., \& Robusto, E. (2013). The gain-loss model: bias and variance of the parameter estimates. Electronic Notes in Discrete Mathematics, 42, 33-40.

de Chiusole, D., \& Stefanutti, L. (2013). Modeling skill dependence in probabilistic competence structures. Electronic Notes in Discrete Mathematics, 42, 41-48.

de Chiusole, D., Stefanutti, L., Anselmi, P., \& Robusto, E. (2013). Assessing parameter invariance in the BLIM: Bipartition models. Psychometrika, 78(4), 710-724. https://doi.org/10.1007/s11336013-9325-5

de Chiusole, D., Stefanutti, L., Anselmi, P., \& Robusto, E. (2015a). Modeling missing data in knowledge space theory. Psychological Methods, 20(4), 506-522.

de Chiusole, D., Stefanutti, L., Anselmi, P., \& Robusto, E. (2015b). Naïve tests of basic local independence model's invariance. The Spanish Journal of Psychology, 18, E26. https://doi.org/10. 1017/sjp.2015.24 
DiBello, L. V., \& Stout, W. (2007). Guest editors' introduction and overview: IRT-based cognitive diagnostic models and related methods. Journal of Educational Measurement, 44(4), 285291.

Doignon, J. P. (1994). Knowledge spaces and skill assignments. In G. Fischer, \& D. Laming (Eds.) Contributions to Mathematical Psychology Psychometrics and Methodology (pp. 111-121). New York: Springer.

Doignon, J. P., \& Falmagne, J. C. (1985). Spaces for the assessment of knowledge. International Journal of Man-Machine Studies, 23, 175-196.

Doignon, J. P., \& Falmagne, J. C. (1999). Knowledge spaces. New York: Springer.

Efron, B. (1979). Bootstrap methods: Another look at the jackknife. The Annals of Statistics, 7, 1-26.

Embretson, S. (1999). Generating items during testing: Psychometric issues and models. Psychometrika, 64(4), 407-433.

Embretson, S. (2005). Measuring human intelligence with artificial intelligence. In R. J. Sternberg, \& J. E. Pretz (Eds.) Cognition and intelligence. New York: Cambridge University Press.

Embretson, S., \& Yang, X. (2006). Automatic item generation and cognitive psychology. Handbook of statistics: Vol. 26 Psychometrics (pp. 747-768). North Holland: Elsevier.

Falmagne, J. C., \& Doignon, J. P. (1988). A class of stochastic procedures for the assessment of knowledge. British Journal of Mathematical and Statistical Psychology, 41, 1-23.

Falmagne, J. C., \& Doignon, J. P. (1988). A Markovian procedure for assessing the state of a system. Journal of Mathematical Psychology, 32, 232-258.

Falmagne, J. C., \& Doignon, J. P. (2011). Learning spaces. New York: Springer.

Gierl, M. J., \& Haladyna, T. M. (2013). Automatic item generation: Theory and practice. Routledge: New York.

Glas, C. A., \& van der Linden, W. J. (2003). Computerized adaptive testing with item cloning. Applied Psychological Measurement, 27(4), 247-261.

Gulliksen, H. (2013). Theory of mental tests. Abingdon: Routledge.

Heller, J. (2017). Identifiability in probabilistic knowledge structures. Journal of Mathematical Psychology, 77, 46-57.

Heller, J., Stefanutti, L., Anselmi, P., \& Robusto, E. (2015). On the link between cognitive diagnostic models and knowledge space theory. Psychometrika, 80(4), 995-1019.

Heller, J., Stefanutti, L., Anselmi, P., \& Robusto, E. (2016). Erratum to: on the link between cognitive diagnostic models and knowledge space theory. Psychometrika, 81(1), 250-251.

Heller, J., \& Wickelmaier, F. (2013). Minimum discrepancy estimation in probabilistic knowledge structures. Electronic Notes in Discrete Mathematics, 42(4), 49-56.
Irvine, S. H., \& Kyllonen, P. C. (2013). Item generation for test development. Abingdon: Routledge.

Junker, B. W., \& Sijtsma, K. (2001). Cognitive assessment models with few assumptions, and connections with nonparametric item response theory. Applied Psychological Measurement, 25, 258272.

Korossy, K. (1999). Modeling knowledge as competence and performance. In D. Albert, \& J. Lukas (Eds.) Knowledge spaces: Theories, empirical research, applications (pp. 103-132). Mahwah: Lawrence Erlbaum Associates.

Robusto, E., Anselmi, P., \& Stefanutti, L. (2010). The gain-loss model: A probabilistic skill multimap model for assessing learning processes. Journal of Educational Measurement, 47(3), 373394.

Rubin, D. B. (1976). Inference and missing data. Biometrika, 63(3), 581-592.

Rudner, L. M. (2010). Implementing the graduate management admission test computerized adaptive test. Elements of adaptive testing (pp. 151-165). New York: Springer.

Schrepp, M. (2005). About the connection between knowledge structures and latent class models. Methodology, 1, 93-103.

Singley, M. K., \& Bennett, R. E. (2002). Item generation and beyond: Applications of schema theory to mathematics assessment. Item generation for test development (pp. 361-384). Mahwah: Lawrence Erlbaum.

Sinharay, S., \& Johnson, M. S. (2013). Statistical modeling of automatically generated items. Automatic item generation: Theory and practice (pp. 183-195). Routledge: New York.

Spoto, A., Stefanutti, L., \& Vidotto, G. (2012). On the unidentifiability of a certain class of skill multi map based probabilistic knowledge structures. Journal of Mathematical Psychology, 56(4), 248-255.

Spoto, A., Stefanutti, L., \& Vidotto, G. (2013). Considerations about the identification of forward-and backward-graded knowledge structures. Journal of Mathematical Psychology, 57(5), 249-254.

Stefanutti, L., Heller, J., Anselmi, P., \& Robusto, E. (2012). Assessing local identifiability of probabilistic knowledge structures. Behavior Research Methods, 44(4), 1197-1211.

Stefanutti, L., \& Robusto, E. (2009). Recovering a probabilistic knowledge structure by constraining its parameter space. Psychometrika, $74,83-96$.

Tatsuoka, K. (1990). Toward an integration of item-response theory and cognitive error diagnosis. In Monitoring skills and knowledge acquisition (pp. 453-488). Lawrence Erlbaum Associates: Hillsdale.

Ünlü, A., Schrepp, M., Heller, J., Hockemeyer, C., Wesiak, G., \& Albert, D. (2013). Recent developments in performance-based knowledge space theory. Knowledge Spaces (pp. 147-192). Berlin: Springer. 